\title{
ERSTE GEMEINSAME ONLINE-SITZUNG DER VÖB-ERWERBUNGS- UND PERIODIKAKOMMISSIONEN
}

\section{von Margret Schmied-Kowarzik und Christoph Genewein}

Zusammenfassung: Am 18. September 2020 fand eine gemeinsame Sitzung der VÖB-Kommission für Periodika und Serienpublikationen mit der VÖB-Kommission für Erwerbungsfragen statt; pandemiebedingt nun erstmals virtuell. Einem Gedankenaustausch über die corona-bedingten Herausforderungen für den bibliothekarischen Arbeitsalltag folgte der Vortrag Wolfgang Mayers über Strategien zur geänderten MwSt. für E-Medien 2020 sowie über die Mehrwertsteuer-Änderungen für gedruckte und elektronische Medien im 2. Halbjahr 2020.

Schlagwörter: VÖB-Kommission für Erwerbungsfragen; VÖB-Kommission für Periodika und Serienpublikationen; virtuelle Sitzung; coronabedingte Herausforderungen; Homeoffice; Mehrwertsteuer

\section{FIRST JOINT ONLINE SESSION OF VÖB ACQUISITION COMMISSION AND VÖB SERIALS COMMISSION}

Abstract: On 18 September 2020, the VÖB Serials Commission and the VÖB Acquisition Commission organized a joint session; due to pandemic reasons it took place online. Main topics were exchanging views about the impact of the COVID-19 pandemic on the librarian's workflows and a lecture on the current VAT's subject matter, given by Wolfgang Mayer.

Keywords: VÖB Acquisition Commission; VÖB Serials Commission; virtual meeting; COVID-19 pandemic; Home office; VAT (Value Added Tax)

DOI: https://doi.org/10.31263/voebm.v73i3-4.5368

(c) Margret Schmied-Kowarzik, Christoph Genewein

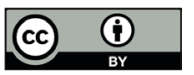

Dieses Werk ist - exkl. einzelner Logos und Abbildungen - lizenziert unter einer Creative-Commons-Lizenz Namensnennung 4.0 International-Lizenz 
Gemeinsame Sitzungen der beiden Kommissionen haben eine lange Tradition.

Am 18. September 2020 fand nun - coronabedingt im Sinne des empfohlenen „Social Distancing“ und aufgrund der erschwerten Reisemöglichkeiten - die erste gemeinsame virtuelle Kommissionssitzung statt.

Wir haben uns für die Verwendung der Software Microsoft Teams entschieden. Ausschlaggebend dafür war, dass diese an der WU Wien lizenziert ist und auch über den Webbrowser ohne vorherige Installation der App verwendet werden kann. Technisch funktionierte das Meeting einwandfrei. Allerdings hatte ein Teilnehmer Schwierigkeiten, sich einzuloggen. Als ratsam hat sich deshalb herausgestellt, vor dem eigentlichen Beginn der Sitzung ein Zeitfenster einzuplanen, um etwaige technische Probleme - insbesondere bei jenen Kolleg*innen, die noch keine Erfahrung mit der betreffenden Applikation haben - zu beheben.

Das Interesse an der Sitzung war groß; eingeloggt waren insgesamt 26 Personen, davon 20 Kommissionsmitglieder und sechs Gäste. An einigen Bibliotheken waren auch mehrere Personen pro Login dabei. Besonders freuten wir uns auch über die Anwesenheit Bruno Bauers, dem VÖB-Präsidenten. Sein Tod am 1. Dezember hat uns schwer getroffen.

Erster Tagesordnungspunkt waren Personalia. Die langjährigen Kommissionsmitglieder Maria Hehle (ULB Tirol/Periodikakommission), Dr. Gerhard Zechner (Vorarlberger Landesbibliothek/Erwerbungskommission) sowie Alfred „Fredi“ Sabitzer (UB Klagenfurt/beide Kommissionen) traten in den wohlverdienten Ruhestand und schieden daher aus den Kommissionen aus. Für ihre langjährige produktive Mitarbeit, für zahlreiche interessante Fachgespräche und angeregte Diskussionen sowie vor allem für ihre Freundschaft danken wir ihnen ausdrücklich.

An ihre Stelle traten drei neue Kolleg*innen: Christina Huggle, BA MA (Vorarlberger Landesbibliothek/Erwerbungskommission), Eva Eberwein (ULB Tirol/Periodikakommission) sowie MMag. Michael Zojer (UB Klagenfurt/beide Kommissionen), die wir herzlich begrüßten.

Der nächste Tagesordnungspunkt war dem Gedankenaustausch über die Stimmung in den einzelnen Einrichtungen während dieser äußerst turbulenten Zeit gewidmet. Besprochen wurden die größten Herausforderungen während des ersten Lockdowns, welche Lehren daraus gezogen wurden und ob Pläne bzw. Richtlinien erarbeitet wurden, sollte sich die Situation wieder verschärfen. Ebenso wurden Veränderungen im Geschäftsgang und im Workflow thematisiert und es wurde die Frage gestellt, ob diese dauerhaft bestehen bleiben sollen. Zu nennen wären etwa die elektronische Rechnungslegung oder der Themenbereich Homeoffice und digitale Arbeit, der 
vielerorts in den Betriebsvereinbarungen verstärkt aufgenommen und spezifiziert wurde. Der Umstieg ins Homeoffice erfolgte überall schnell. Teilweise wurden auch Arbeitsprozesse umgestellt, die nach dem Lockdown beibehalten wurden (beispielsweise Hochladen von Rechnungs-Scans in Alma im WU-Zeitschriftenmanagement). Einhelliger Tenor war, dass die Nachfrage nach elektronischen Medien gestiegen ist und das elektronische Angebot in einigen Institutionen vielfach massiv ausgeweitet wurde. In den Nutzungsstatistiken konnte festgestellt werden, dass das bisher bestehende Angebot verstärkt genutzt wurde und auch gegenüber E-Medien bisher eher reservierte Benutzer*innengruppen verstärkt auf sie zurückgriffen. Dies hatte jedoch auch zur Folge, dass der Arbeitsaufwand weiter gestiegen ist. Beispielsweise zeigte sich nun verstärkt das Problem, dass zahlreiche Einzel-E-Books nicht als Lizenz für Bibliotheken erwerbbar sind, die Aufklärungsarbeit darüber war mit einem Mehraufwand verbunden. Wo der Benutzer*innenbedarf gedeckt werden konnte, entstanden im Buchbereich teilweise auch höhere Kosten, da insbesondere bei kleinen Verlagshäusern E-Books gegenüber gedruckten Büchern unverhältnismäßig teuer sind.

Den Hauptteil der Sitzung bildete der dritte Tagesordnungspunkt zum Thema Mehrwertsteuer. Diese überaus komplexe Steuerproblematik referierte Wolfgang Mayer von der UB Wien, der eine fundierte Darstellung bot.

Der erste Teil des Vortrags war den „Strategien zur geänderten MwSt. für e-Medien 2020" gewidmet; der zweite den Mehrwertsteuer-Änderungen für gedruckte und elektronische Medien im 2. Halbjahr 2020.

Ausgehend von den rechtlichen Rahmenbedingungen - Richtlinien der Europäischen Union und dem Steuerreformgesetz 2020 - präsentierte Wolfgang Mayer das von ihm gemeinsam mit Frank Koren-Wilhelmer, Brigitte Kromp, Georg Fessler und Bruno Bauer im Rahmen der KEMÖ AG „SteuerRefG 2020 elektronische Publikationen“ erarbeitete Papier „Produktgruppeneinteilung von E-Ressourcen für Mehrwertsteuerberechnung", wobei die erste Version aus dem Dezember 2019 und die revidierte Fassung aus dem September 2020 stammt. Bei diesem Dokument handelt es sich um eine Expert*inneneinschätzung (und nicht um ein rechtsverbindliches Gutachten), die als Leitfaden für die steuerliche Bewertung von elektronischen Medien herangezogen werden kann und mit ihrer Einteilung von E-Ressourcen in elf Produktcluster als Orientierungshilfe dienen soll.

Davon abgesehen werden alle bisher mit $10 \%$ besteuerten Medien ab dem 2. Halbjahr 2020 lediglich mit $5 \%$ MwSt. belastet.

Bei der anschließenden regen Diskussion wurde vor allem über die Produktcluster 6 - mit Volltexten angereicherte Datenbanken - und 7 - originäre Volltextdatenbanken - debattiert. 
Thematisiert wurde der Mehraufwand, der für die Bibliotheken aber auch für Lieferanten durch die kurzfristigen Änderungen der Besteuerung und deren komplexe rechtliche Regelung entstand.

Die Kommissionsvorsitzenden und auch die Teilnehmer*innen bedankten sich beim Vortragenden für seine profunde Darstellung dieses komplizierten Sachverhaltes und für die Beantwortung der zahlreichen Fragen.

Einhellig begrüßt wurde auch das Online-Format der Sitzung, das zwar kein vollwertiger Ersatz für ein persönliches Treffen, jedoch eine probate Lösung bietet, um trotz der pandemiebedingten Schwierigkeiten weiter in Kontakt zu bleiben und produktiv österreichweit zusammenzuarbeiten.

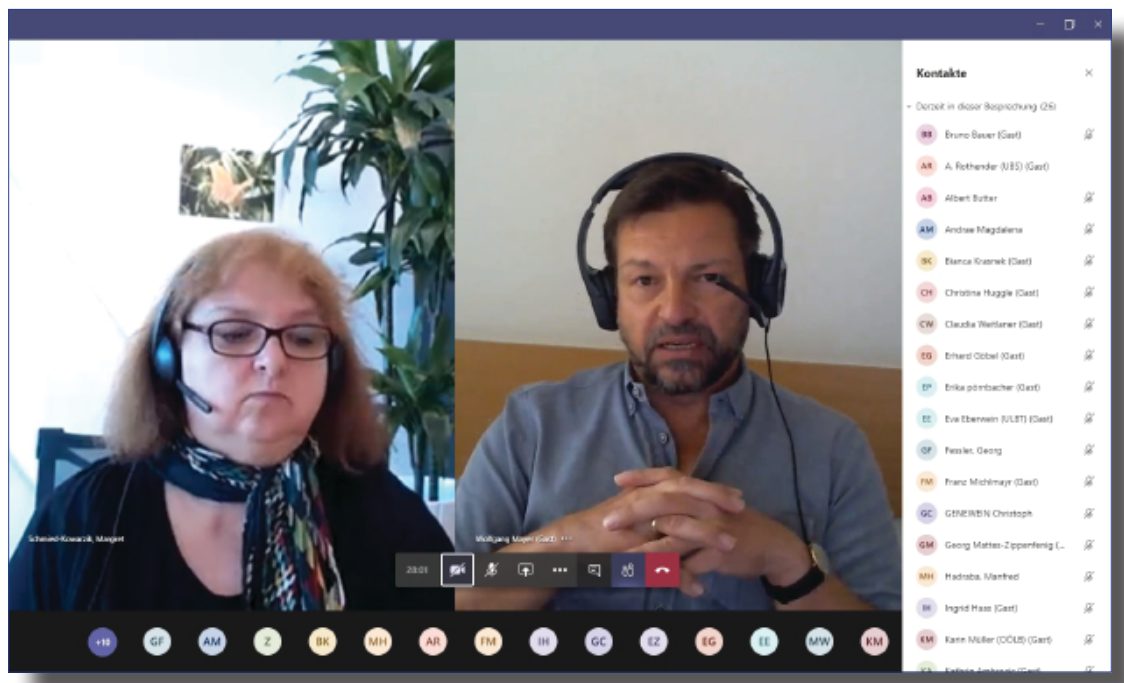

Abb.: Screenshot der Sitzung, angefertigt von Bruno Bauer ${ }^{\dagger}$ (links: Margret Schmied-Kowarzik, rechts: Wolfgang Mayer)

Mag. ${ }^{a}$ Margret Schmied-Kowarzik Wirtschaftsuniversität Wien, Universitätsbibliothek E-Mail: margret.schmied-kowarzik@wu.ac.at

Mag. Christoph Genewein AK Bibliothek Wien für Sozialwissenschaften E-Mail: christoph.genewein@akwien.at 